\title{
Analysis of Gait Dynamics of ALS Disease and Classification of Artificial Neural Networks
}

\author{
Omer AKGUN, Aydin AKAN, Hasan DEMIR, Tahir Cetin AKINCI
}

\begin{abstract}
In this study, a gait device was used for gathering data. A group comprising control group and ALS patients was requested to walk using this device. Gait signals of the control group individuals and ALS patients taken from their left feet were recorded by means of the sensors sensitive to the force which was placed to the device. Spectral and statistical analyses of these signals were made. The results obtained from these analyses were used for making classification with Artificial Neural Network. In consequence of the classification, the individuals with ALS disease were diagnosed accurately with an average rate of $82 \%$. In the study, the signals taken from left foot of 14 normal individuals and 13 ALS patients were analyzed.
\end{abstract}

Keywords: ALS Disease; Artificial Neural Nets; Gait Dynamics Analysis; Piezo Electric Sensors; Sound and Vibration

\section{INTRODUCTION}

Amyotrophic lateral sclerosis (ALS) is a disease characterized by the specific death of brain neurons in the brain stem, brain stem and spinal cord. As a result of neuronal death, voluntary muscles in the body become weak and weakness in voluntary muscles results in a general paralysis. Generally, these patients are not lost in sexual, cognitive, and sensory functions. Muscle weakness in the hands, in the laces, in the mouth-pharyngeal region or on the underside spreads continuously. In $75 \%$ of patients, first symptoms were seen in arm and leg muscles. As a result of the statement of ALS, whichever muscle is affected in the body, that part is first damaged $[1,2]$.

Nowadays, the progress in technological means provides the widespread usage of walking analyses with the purpose of diagnosis and treatment planning and also measuring and following of the treatment in research and clinical applications on musculo skeletal system. Gait analysis takes an important place particularly in diagnosis and treatment of musculo skeletal system problems which are observed on neuromuscular, brain, spinal cord, peripheral nerve, muscular, bone and joint diseases. To perform these tests, walking tests must be made systematically by means of the suitable measurement tools.

The studies made on Gait go back to Aristo period [3]. Borelli, and in the $19^{\text {th }}$ century, Marey made dynamic studies concerning human walking [4, 5]. Edweard Muybridge, a photographer, contributed to gait analysis considerably by means of making researches through photographs. In 1895, Braune and Fisher put forward a new approach by transforming available photo images into numeric variables. Eberhart and Inman expanded on these experiments using a perforated disc turning in front of the camera objective and obtained successful results. The foundations of gait analysis and its clinical applications which have still been applied were laid by Verne Inman and Jacquelin Perry [3-6].

Nowadays, the clinical and biomedical researches made on gait analysis comprehend evaluation, description and interpretation of gait numerically. Even though this diagnosis could be understood through visual examinations made by experienced physicians, gait analysis technology is a requirement to prove the problem numerically, to record the data obtained for revaluation and to reveal the efficiency of the treatment objectively. The following order is followed during these tests.

At Modern walking analysis laboratories, firstly, the patient's walk is evaluated visually and by video records. The movement data are gathered and transferred to computer by means of the sensors tied to the determined points of the patient. In addition to these data, at developed laboratories, the patients' dynamic electromyography and energy consumption measurements are also performed. These data obtained are transformed into numerical data at data system by means of special computer software. Lastly, the diagnosis is concluded by joint evaluation of both the data analysis and the patient's clinical status [3-7].

This study focused on kinetical analysis of gait dynamics, and the sole datum which could be measured at analysis is the ground reaction force vector (GRFV). GRFV is measured by means of the pressure-sensitive plaques called force plate which measures the total force the foot applied on the ground. The transducers which are placed to the side, front, back and inside the plate measure the components on each three planes climbing on the force plate level, and transfer this datum to the computer [3, 8 , 9]. Besides, in the study, walking signals were recorded through placing the piezoelectric sensors sensitive to force under the foot.

\section{MOTOR NEURON DEGENERATION ALS DISEASE}

Amyotrophic Lateral Sclerosis (ALS) is a progressive neurodegenerative disease. This disease may spread to whole body by affecting motor nerves. At first, its symptoms are mild, this condition makes its recognition difficult, and it can progress even to paralysis at further phases. This disease does not affect mental abilities. Beginning of this disease called Charcot in many countries goes back to 1840 by Aran Duchenne [3-5].

Progress of ALS disease comprises three phases; at first, it reveals itself by some mild symptoms as fatigue, muscle pains, weakness at arms and legs; in the second phase, the patient begins facing the problems such as indigestion, breathing problems. At the final phase, the patient is paralyzed. [4-6].

ALS is a disease in which adult type motor neuron diseases are observed most frequently. Both upper motor neuron (UMN) and lower motor neuron (LMN) involvement can occur. At ALS disease, muscle weakness 
starts locally and spreads to neighbouring muscles quickly. In studies, it has been seen that the disease mostly started on arms, however in a number of cases the disease started on legs. $25 \%$ of patients have bulber (involvement of subcranial nerves). At LMN involvement, there is weakness of hand movements and dropping of foot, at UMN involvement, lumbering, balance-coordination disorder and fatigue are the first symptoms. In this study, the walking test results used for the diagnosis of ALS disease by physicians were analysed by means of using mathematical and signal processing techniques [7-14].

\section{ALS DATA ACQUISITION SYSTEM}

By means of using piezoelectric sensors sensitive to force under the foot, 60-second gait signals were recorded from 14 Normal and 13 ALS patients. These signals were transformed into 18000 series $x(n)$ discrete signals and transferred to computer environment. These signals were taken from physionet.org web site [15]. These signs are from the physioNet.org website. This site is the reference source for medical signs. And it also does not require an ethics committee report.

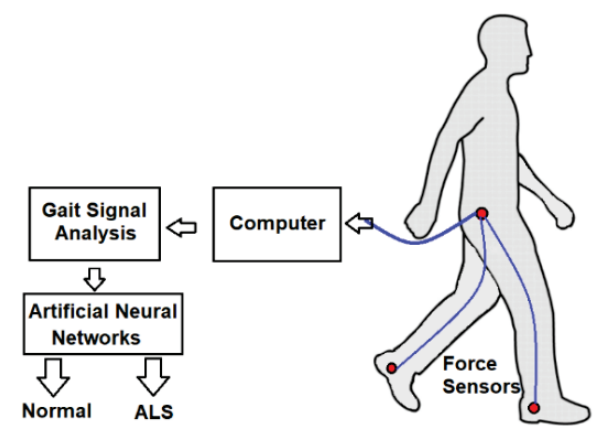

(a)

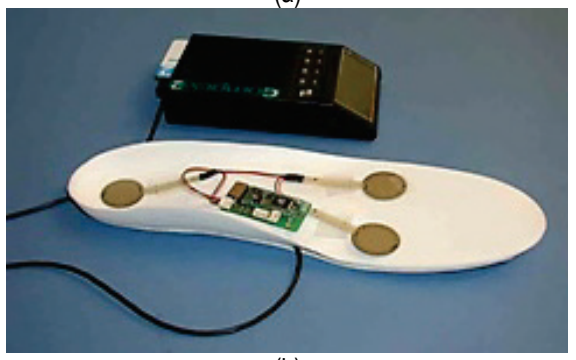

(b)

Figure 1 Fault tree of rule A: (a) Schematic data collection system, (b) Force sensor device

\section{MODELLING BY TIME-AMPLITUDE AND TIME- FREQUENCY ANALYSIS}

In this analysis also called Spectral Analysis, it is supposed that the datum in Time/Space environment comprises the total of different sinusoids while being transferred to frequency environment. The amplitudes and phases of these sinusoidal waves are determined. The functions which are obtained by means of transferring the datum from time/space environment to frequency environment are called spectrum. Here, it may be described that Amplitude spectrum is the indication of amplitude information of sinusoidal within the Amplitude Spectrum signal as to the frequency axis; on the other hand, the Phase Spectrum is an indication of starting angles of sinusoidal within the signal as to the frequency axis. Because the signals in Time domain are real, Amplitude spectrums are symmetric; if a signal is symmetric in time period, its equivalent will be real in frequency domain $[14,16,17]$.

$$
\begin{aligned}
& x(\Omega)=\int_{-\infty}^{+\infty} x(t) \mathrm{e}^{-j \Omega t} \mathrm{~d} t \\
& x(\Omega)=\frac{1}{2 \pi} \int_{-\infty}^{+\infty} x(t) \mathrm{e}^{-j \Omega t} \mathrm{~d} \Omega
\end{aligned}
$$

If Fourier serial is extended to non-periodical signals, Fourier transformation is obtained. This transformation is a generalized shape of Fourier series. By means of this transformation, the period of a non-periodical signal can be considered as infinite. Basic frequency of a signal whose period is infinite goes to zero in limit. Transformation equivalents are given in Eq. (1) and Eq. (2).

Gait signals can be modelled as signals changing by time as in Eq. (3). In Eq. (3), c(n) shows the amplitude of walking signal, $f_{1}(n)$ instantaneous frequency, $w(n)$ white noise $[16,17]$.

$$
\begin{aligned}
& x(n)=c(n) \cdot \cos \left(2 \pi \sum_{\lambda=-\infty}^{n} f_{i}(\lambda)\right)+w(n) \\
& 0<n<N-1 \quad(N=18000)
\end{aligned}
$$

Amplitude and phase spectrums of gait signals $x(n)$ are stated by Eq. (4). Besides, Discrete Fourier Transform (DFT) is applied on $x(n)$ signals, DFT equation is given in Eq. (5) and Eq. (6) $[13,14,16-18]$. Here, $\Delta \omega=2 \pi / N$ is sampling point (step) at frequency domain.

$$
\begin{aligned}
& X[k]=\sum_{n=0}^{N-1} x(n) \mathrm{e}^{-j \frac{2 \pi}{N} k n}, k=0,1, \ldots, N-1 \\
& X[k]=\sum_{n=0}^{N-1} x[k] \mathrm{e}^{-j \frac{2 \pi}{N} k n}, k=0,1, \ldots, N-1 \\
& X[k]=\frac{1}{N} \sum_{n=0}^{N-1} X[k] \mathrm{e}^{-j \frac{2 \pi}{N} k n}, n=0,1, \ldots, N-1
\end{aligned}
$$

\subsection{Calculation of Statistical Parameters}

Several methods are used in calculation of statistical parameters of signed based analyses. In this study, two fundamental parameters are the mean value, and the standard deviation. For a given data set $\left\{x_{i}\right\}$ these are defined as the following Eqs. (7) and (8).

$$
\begin{aligned}
& \mu=\frac{1}{N} \sum_{i=1}^{N} x_{i} \\
& \sigma=\sqrt{\frac{1}{N} \sum_{i=1}^{N}\left(x_{i}-\mu\right)^{2}}
\end{aligned}
$$

where $N$ is the number of the data points. These statistical parameters may be used to perform a quick check of the changes in the statistical behaviour of a signal [20]. 


\subsection{Artificial Neural Networks (ANN)}

Artificial Neural Networks (ANN) is a mathematical approach, which is developed to make human brain realize the abilities as deriving and discovering new information by learning, without any help, automatically. The studies on ANN in consequence of mathematical modelling of human brain learning process firstly started with modelling of neurons as biological units forming the brain and their applications in computer systems, afterwards, in parallel with the progress of computer systems, they became usable in many fields. Even if the systems working with ANN are accepted pretty slower than the human brain in terms of decision speed, they are very fast by their abilities to distinguish. ANNs are comprised of many cells and these cells can perform all complicated functions easily by means of working simultaneously [21-23].

$$
\begin{aligned}
& y=f\left(\sum_{j=1}^{n} w_{j} x_{j}-u\right) \\
& f(x)=\frac{1}{1+\mathrm{e}^{-\beta x}}
\end{aligned}
$$

Each neuron makes intermittent sum of $x$ input vector comprising $n$ element and constitutes output value by activation function. In Eq. (9), $f($.) shows activation function, $w_{j}$ interval coefficient of input vector for $j$ element. Activation function is given by Eq. (10). In this study, a basic ANN architecture was used comprising 3 hidden layers without feedback (Fig. 2).

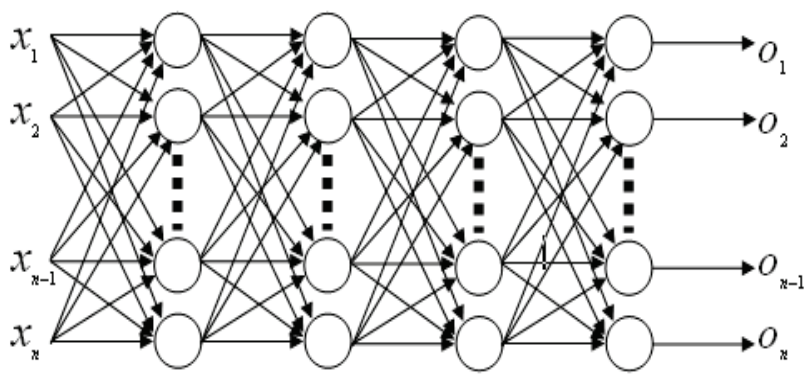

Figure 2 The structure of a basic ANN with feed-forward and 3 hidden layers

\section{APPLICATION TO THE SIGNALS}

Instantaneous energy calculated at walking signals is absolute value of amplitude square of the signals. Changes by time for each Normal and pathological signals belonging to ALS data were given in Fig. 3. Besides, in Tab. 1, maximum, minimum amplitudes and energy values of signals taken from left feet of 14 Normal individuals and 13 ALS patients were given.

When Fig. 3 and Tab. 1 were examined, it was observed that the values at maximum interval mostly took 0 and 0.1 values, whereas minimum values took -0.6 values at ALS signals. At Normal signals, the values at maximum interval took larger values, whereas the values at minimum interval took -0.6 and lower values. ALS signals have lower energy levels than Normal signals. In Fig. 4, amplitude and phase spectrums of Normal and ALS walking signals are given.

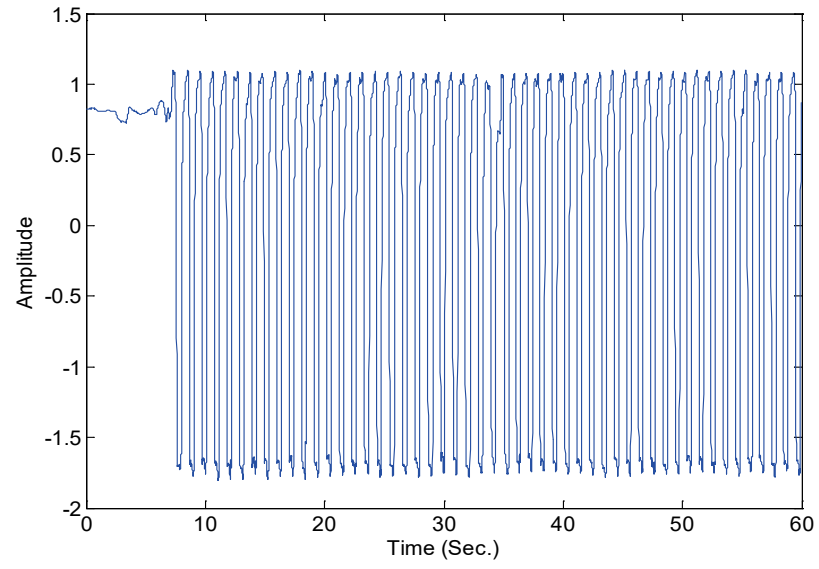

(a)

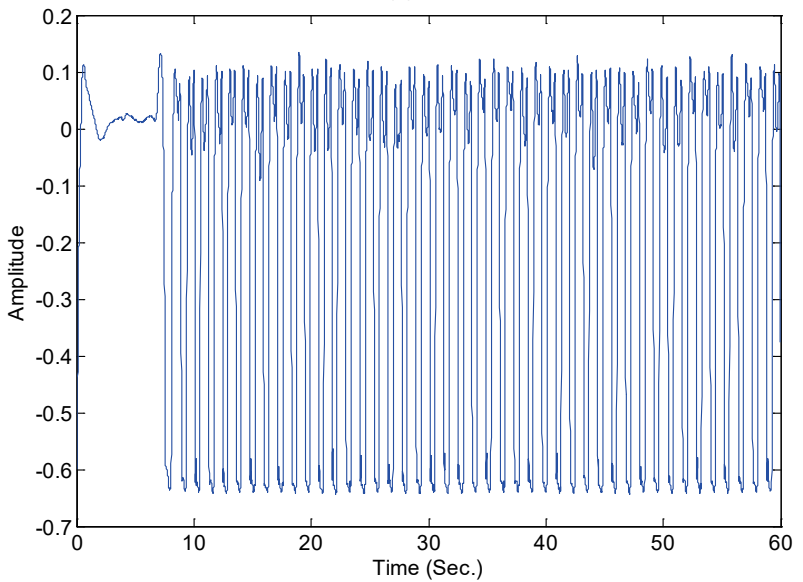

(b)

Figure 3 Time-Amplitude graphics of Normal (upper) and ALS (lower) left foot walking signals: (a) Normal, (b) ALS

Table 1 Max./min. and energy values of left foot walking signals of 14 Normal

\begin{tabular}{|c|c|c|c|c|c|c|}
\hline \multirow{2}{*}{ No } & \multicolumn{2}{|c|}{ Normal } & \multicolumn{2}{|c|}{ ALS } & \multirow{2}{*}{$\begin{array}{l}\text { Normal } \\
\text { energy }\end{array}$} & \multirow{2}{*}{$\begin{array}{c}\text { ALS } \\
\text { energy }\end{array}$} \\
\hline & $\max$. & min. & $\max$. & $\min$. & & \\
\hline 1 & 0.3 & -0.6 & 0 & -0.6 & 2257 & 2582 \\
\hline 2 & 0.2 & -0.6 & 0.2 & -0.5 & 2774 & 1508 \\
\hline 3 & 0 & -0.6 & 0.1 & -0.6 & 2958 & 2584 \\
\hline 4 & 0 & -0.6 & 0 & -0.5 & 3147 & 1201 \\
\hline 5 & 0.7 & -2 & 0.1 & -0.6 & 26154 & 1997 \\
\hline 6 & 0.2 & -2 & -0.1 & -0.6 & 27961 & 2816 \\
\hline 7 & 0.1 & -0.6 & 0.1 & -0.6 & 2665 & 2163 \\
\hline 8 & 0.5 & -2 & 0 & -1.6 & 26400 & 23085 \\
\hline 9 & 1 & -1.7 & 0.2 & -0.6 & 25710 & 2850 \\
\hline 10 & 0.8 & -1.8 & 0 & -0.6 & 26216 & 2587 \\
\hline 11 & 0.4 & -2 & 0.1 & -0.6 & 25925 & 2356 \\
\hline 12 & 0.2 & -0.6 & 0 & -0.6 & 2573 & 2605 \\
\hline 13 & 0.5 & -2 & 0 & -0.6 & 23750 & 2442 \\
\hline 14 & 0.3 & -0.6 & & & 2656 & \\
\hline
\end{tabular}
individuals and $13 \mathrm{ALS}$ patients

When Fig. 4 and Tab. 2 were examined, it was determined that ALS signals had spectral amplitude values around $1 \mathrm{~Hz}$ and these values were smaller than normal signals. It has been seen that slopes of ALS signals are smaller than Phase spectrum and table values. Also, one of the special values that can define characteristic distribution of $x(n)$ walking signals is its expected values and variances.

When Tab. 3 was examined, it was determined that mean values of ALS gait signals usually took values around -0.2 , and Normal signals took smaller mean values. At another examination made according to the variance values, it was determined that ALS signals had pretty smaller values than normal signals. 


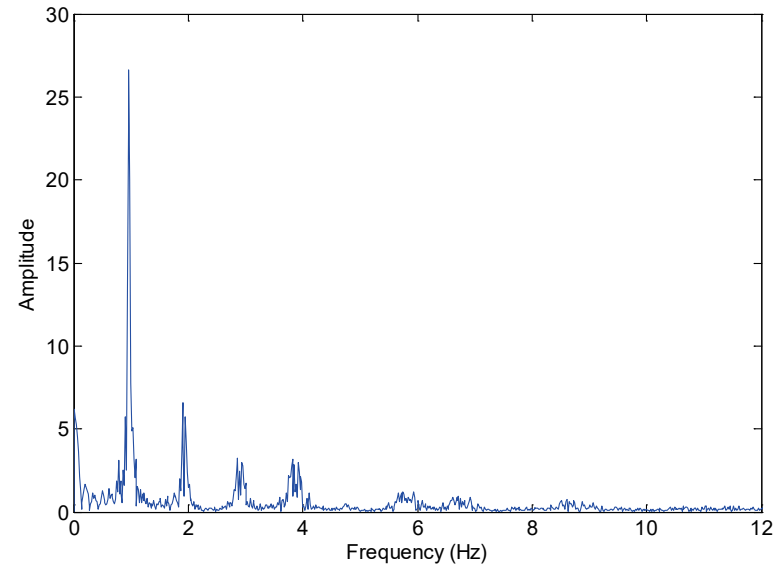

(a)

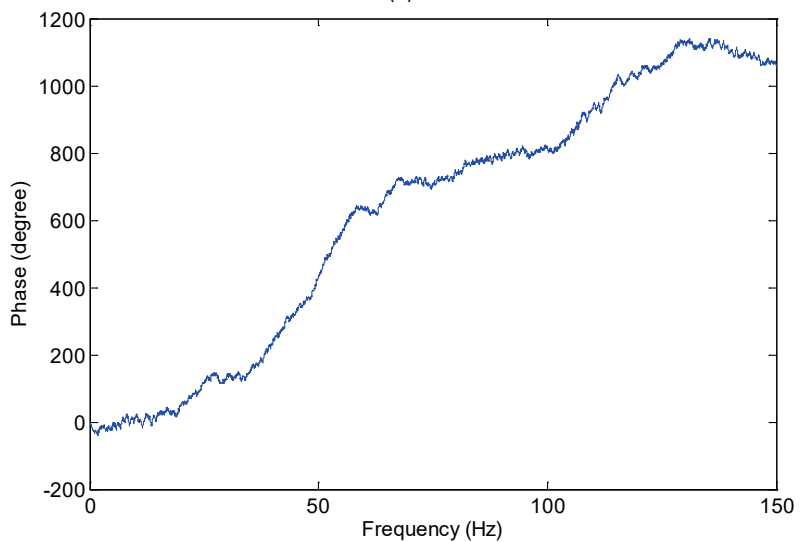

(b)

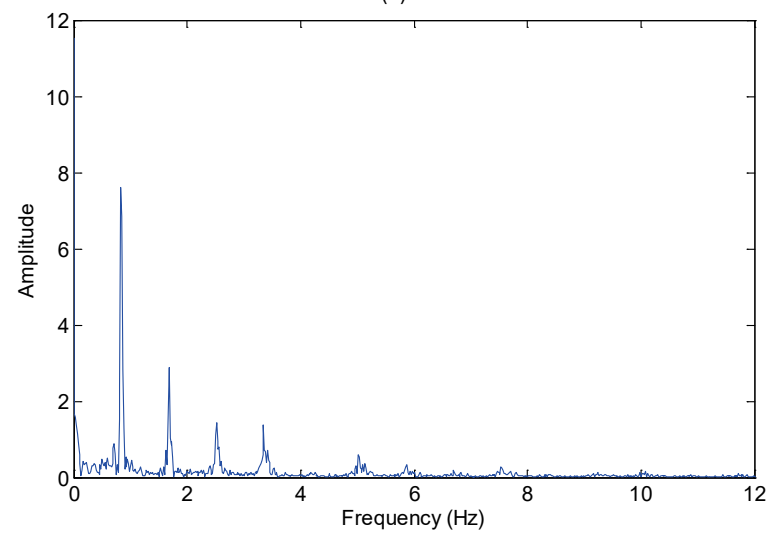

(c)

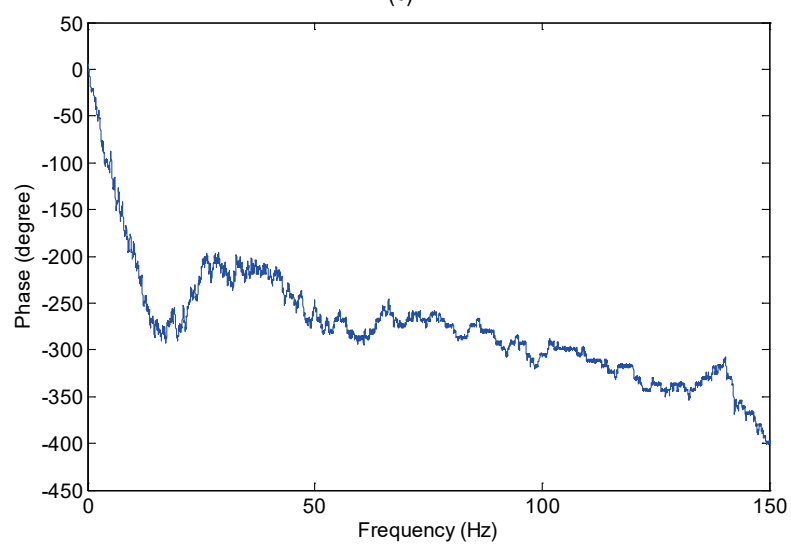

(d)

Figure 4 Amplitude and phase spectrums of Normal (upper) and ALS (lower) signals: (a) and (b) Normal, (c) and (d) ALS.
Table 2 Spectral amplitude values of Normal and ALS signals around $1 \mathrm{~Hz}$ and max./min. degrees of phase spectrums

\begin{tabular}{|c|c|c|c|c|c|c|}
\hline \multirow{2}{*}{ No } & \multirow{2}{*}{$\begin{array}{c}\text { Normal } \\
\text { amplitude }\end{array}$} & \multirow{2}{*}{$\begin{array}{c}\text { ALS } \\
\text { amplitude }\end{array}$} & \multicolumn{2}{c|}{ Normal } & \multicolumn{2}{c|}{ ALS } \\
\cline { 5 - 7 } & 10.2 & 3.6 & 1134 & -180 & 1339 & -48 \\
\hline 1 & 12.4 & 8.3 & 7 & -459 & 1600 & -235 \\
\hline 2 & 7.8 & 9.6 & 100 & -114 & 670 & -35 \\
\hline 3 & 4.2 & 534 & -35 & 829 & 0 \\
\hline 4 & 6.1 & 4.7 & 12 & -500 & 645 & -32 \\
\hline 5 & 39.2 & 4 & 311 & -46 & 802 & -41 \\
\hline 6 & 21.5 & 5.7 & 3049 & -20 & 350 & -28 \\
\hline 7 & 79 & 8.7 & 1221 & 0 & 395 & -84 \\
\hline 8 & 26.3 & 9.9 & 1072 & -31 & 261 & -91 \\
\hline 9 & 26.6 & 8.6 & 289 & -102 & 529 & -136 \\
\hline 10 & 30.2 & 7.6 & 1952 & -93 & 0 & -400 \\
\hline 11 & 22.9 & 9 & 1081 & -200 & 17 & -42 \\
\hline 12 & 8.7 & 6.5 & 455 & -154 & 204 & -89 \\
\hline 13 & 21.6 & & 603 & -74 & & \\
\hline 14 & 73 & & & & & \\
\hline
\end{tabular}

Table 3 Mean values and variances of Normal and ALS walking signals

\begin{tabular}{|c|c|c|c|c|}
\hline $\begin{array}{c}\text { Patients } \\
\text { No }\end{array}$ & Control mean & $\begin{array}{c}\text { ALS } \\
\text { mean }\end{array}$ & $\begin{array}{c}\text { Control } \\
\text { variance }\end{array}$ & $\begin{array}{c}\text { ALS } \\
\text { variance }\end{array}$ \\
\hline 1 & -0.062 & -0.323 & 0.121 & 0.038 \\
\hline 2 & -0.128 & -0.018 & 0.137 & 0.083 \\
\hline 3 & -0.308 & -0.234 & 0.069 & 0.088 \\
\hline 4 & -0.323 & -0.151 & 0.069 & 0.043 \\
\hline 5 & -0.461 & -0.234 & 1.240 & 0.055 \\
\hline 6 & -0.858 & -0.341 & 0.816 & 0.040 \\
\hline 7 & -0.259 & -0.197 & 0.080 & 0.081 \\
\hline 8 & -0.682 & -1.055 & 1.001 & 0.168 \\
\hline 9 & 0.00007 & -0.211 & 1.428 & 0.113 \\
\hline 10 & -0.267 & -0.281 & 1.385 & 0.064 \\
\hline 11 & -0.694 & -0.191 & 0.958 & 0.094 \\
\hline 12 & -0.117 & -0.268 & 0.129 & 0.072 \\
\hline 13 & -0.528 & -0.236 & 1.040 & 0.079 \\
\hline 14 & -0.089 & & 0.139 & \\
\hline
\end{tabular}

Table 4 Spectral amplitude values of Normal and ALS signals around $1 \mathrm{~Hz}$ and max./min. degrees of phase spectrums

\begin{tabular}{|c|l|c|c|}
\hline $\begin{array}{c}\text { Number of hidden } \\
\text { layers of ANN }\end{array}$ & $\begin{array}{c}\text { Number of neurons in } \\
\text { hidden layers }\end{array}$ & $\begin{array}{c}\text { Success } \\
\text { ratio } \\
\text { (Logsig) }\end{array}$ & $\begin{array}{c}\text { Success } \\
\text { Ratio } \\
\text { (Tansig) }\end{array}$ \\
\hline 1 & $(4)$ & 0.6875 & 0.9375 \\
\hline 1 & $(8)$ & 0.8125 & 0.8125 \\
\hline 1 & $(16)$ & 0.8750 & 0.8125 \\
\hline 2 & $(10)-(5)$ & 0.8125 & 0.8750 \\
\hline 2 & $(8)-(4)$ & 0.8125 & 0.6250 \\
\hline 2 & $(6)-(5)$ & 0.8750 & 0.6875 \\
\hline 3 & $(5)-(3)-(1)$ & 0.8750 & 0.9375 \\
\hline 3 & $(15)-(8)-(7)$ & 0.8125 & 0.8750 \\
\hline 3 & $(10)-(9)-(8)$ & 0.6875 & 0.8125 \\
\hline 4 & $(16)-(14)-(12)-(8)$ & 0.8750 & 1.0000 \\
\hline 4 & $(10)-(10)-(10)-(10)$ & 0.6250 & 0.7500 \\
\hline 4 & $(8)-(7)-(6)-(5)$ & 0.8750 & 0.8125 \\
\hline
\end{tabular}

The biggest and the smallest amplitude, energy values, mean value, variances of gait signals, the biggest and the smallest values of amplitude and phase spectrums were given as input to feed forward ANN. At neurons, sigmoidal and tangent sigmoidal functions were used as activation function. 45 data were used as control group, and 16 data as test group. Artificial Neural Network was trained by a back propagating error algorithm, and mean-square error value on training is given in Eq. (11).

$$
E=\frac{1}{N} \sqrt{\sum_{i=1}^{N}\left(y_{i}-d_{i}\right)^{2}}
$$

In this expression, $\mathrm{N}$ value shows 7 values obtained for each trained vector, $y_{i}$ shows output value for $i$. input value 
of Artificial Neural Network, $d_{i}$ shows target value for $i$. input datum. At the classification made by ANN using these data, performances of methods are given in Tab. 4 .

\section{CONCLUSION}

At time-amplitude analysis, maximums of ALS gait signals take smaller values. A deviation towards decrease at normal signals may be seen from -0.6 unit determined as a shared value at Minimum values. ALS signals stand out by small energy values. At amplitude spectrum, amplitude values of ALS walking signals around $1 \mathrm{~Hz}$ transparently differentiate with their smaller amplitudes. At phase spectrum, phase curves of ALS walking signals reveal themselves by their small slopes. ALS signals take mean values around -0.2 . Besides, it was determined that pretty small values of variances were distinctive features peculiar to ALS signals. At a classification made by ANN using all analysis values, ALS disease was diagnosed accurately with an average rate of $82 \%$.

\section{REFERENCES}

[1] Firincioglu, M., Yavuz, B., \& Koc, F. (2016). Anterior Horn Cell Diseases. Archives Medical Review Journal, 25(4), 269-303.

[2] Tiryaki, E. \& Horak, A. H. (2014). ALS and other motor neuron diseases. Continuum (Minneap Minn), 20, 1185 1207. https://doi.org/10.1212/01.CON.0000455886.14298.a4

[3] Akgun, O., Demir, H., Akan, A., \& Akinci T. C. (2008). Detection of ALS Disease Using AR Models of Gait Dynamics. Eleco 2008 Conference on Electrical, Electronics and Computer Engineering / Bursa.

[4] Bedlack, R. S. \& Aggarwal, S. (2009). ALS update: Signs of progress, reasons for hope. Continuum Lifelong Learning Neurology, 15(1), 83-110. https://doi.org/10.1212/01.CON.0000300009.02717.a2

[5] Rowin, J. (2009). Approach to the patient with suspected: A clinical's guide. Continuum Lifelong Learning Neurology, 5(1), 13-34. https://doi.org/10.1212/01.CON.0000300006.49351.2c

[6] Lacomblez, L., Bensimon, G., Leigh, P. N., Guillet, P., \& Meininger, V. (1996). Dose-ranging Study of Riluzole in Amyotrophic Lateral Sclerosis. Amyotrophic Lateral Sclerosis/Riluzole Study Group II. Lancet, 347(9013), 14251431. https://doi.org/10.1016/S0140-6736(96)91680-3

[7] Mazzini, L., Mareschi, K., Ferrero, I., Vassallo, E., Oliveri, G., Nasuelli, N., Oggioni, G. D., Testa, L., \& Fagioli, F. (2008). Stem cell treatment in Amyotrophic Lateral Sclerosis. J. Neurol Sci., 265(1-2), 78-83. https://doi.org/10.1016/j.jns.2007.05.016

Mazzini, L., Mareschi, K., Ferrero, I., Vassallo, E., Oliveri, G., Boccaletti, R., Testa, L., Livigni, S., \& Fagioli, F. (2006). Autologous mesenchymal stem cells: clinical applications in amyotrophic lateral sclerosis. Neurol. Res., 28(5), 523-526. https://doi.org/10.1179/016164106X116791

[8] Martinez, H. R., Gonzalez-Garza, M. T., Moreno-Cuevas, J. E., Caro, E., Gutierrez-Jimenez, E., \& Segura, J. J. (2009). Stem-cell transplantation into the frontal motor cortex in amyotrophic lateral sclerosis patients. Cytotherapy, 11(1), 26-34. https://doi.org/10.1080/14653240802644651

[9] Eisen, A. (2009). Amyotrophic lateral sclerosis: A 40-year personal perspective. Clin. Neurosci, 16(4), 505-512. https://doi.org/10.1016/j.jocn.2008.07.072
[10] Appel, S. H., Engelhardt, J. I., Henkel, J. S., Siklos, L., Beers, D. R., \& Yen, A. A. (2008). Hematopoietic stem cell transplantation in patients with sporadic amyotrophic lateral sclerosis. Neurology, 71(17), 326-34. https://doi.org/10.1212/01.wnl.0000327668.43541.22

[11] www.alsmndalliance.org

[12] Fattah, S. A., Iqbal, A., Jumana, M. A. (2012). Identifying the Motor Neuron Disease in EMG Signal Using Time and Frequency Domain Features with Comparison. Signal \& Image Processing, an International Journal (SIPIJ), 3(2), 99-114.

[13] Kehri, V., Ingle, R., \& Awale, R. (2017). Technique of EMG Signal Analysis and Classification of Neuromuscular Diseases. Advances in Intelligent Systems Research, 137, 485-491. https://doi.org/10.2991/iccasp-16.2017.71

[14] https://www.physionet.org/

[15] Vaseghi, V. S. (1996). Advanced Signal Processing and Digital Noise Reduction. John Wiley, New York. https://doi.org/10.1007/978-3-322-92773-6

[16] Lekshmi, S. S., Selvam, V., \& Pallikonda Rajasekaran, M. (2014). EEG signal Classification using Principle Component Analysis and Wavelet Transform with Neural Network. International conference on communication and signal processing, IEEE conference, 1, April 3-5, 2014, 456459.

[17] Taylor, J. K. (1990). Statistical Techniques for Data Analysis, Lewis Publishers.

[18] Karlsson, S., Yu, J., \& Akay, M. (2000). Time-frequency Analysis of Myoelectric Signals during Dynamic Contractions: a comparative study. Biomedical Engineering, IEEE Transactions on, 47(2), 228-238. https://doi.org/10.1109/10.821766

[19] Englehart, K., Hudgins, B., Parker, P. A., \& Stevenson, M. (1999). Classification of the Myoelectric Signal Using Time-Frequency Based Representations. Med. Eng. Phys. 21, 431-438. https://doi.org/10.1016/S1350-4533(99)00066-1

[20] Haykin, S. (1994). Neural Networks: A Comprehensive Foundation, Mac Millan College Publishing Co., New York.

[21] Jain, A. K., Mao, J., \& Mohiuddin, K. (1996). Artificial Neural Networks: Tutorial. IEEE Computer Special Issues on Neural Computing, 29(3), 56-63. https://doi.org/10.1109/2.485891

[22] Gratwicke, J., Jahanshahi, M., \& Foltynie, T. (2015). Parkinson's Disease Dementia: A Neural Networks Perspective. Brain - A Journal of Neurology, 138(6), 14541476. https://doi.org/10.1093/brain/awv104

\section{Contact information}

Omer AKGUN, Assist. Prof.

Marmara University, Department of Computer Engineering,

Kuyubasi, Istanbul, 34722, Turkey

oakgun@marmara.edu.tr

\section{Aydin AKAN, Prof}

Katip Celebi University, Subdiscipline of Biomedical Engineering,

Avcilar, Izmir, 35620, Turkey

aydin.akan@ikc.edu.tr.

Hasan DEMIR, Assist. Prof.

Namik Kemal University, Electronics \& Communications Engineering,

Tekirdag, 59000, Turkey

hdemir@nku.edu.tr

Tahir Cetin AKINCI, Assoc. Prof.

Istanbul Technical University, Department of Electrical Engineering,

Maslak, Istanbul, 34320, Turkey

akincitc@itu.edu.tr 